ACTA UNIVERSITATIS LODZIENSIS

Folia Litteraria Romanica 15, 2020

https://doi.org/10.18778/1505-9065.15.14

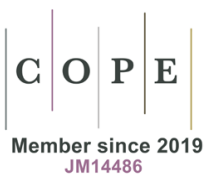

\author{
Anita Staroń \\ Université de Lodz \\ iD ORCID ID : 0000-0002-4968-885X \\ anita.staron@uni.lodz.pl
}

\title{
La notion de mépris dans la critique d'art de Baudelaire et de Mirbeau
}

\begin{abstract}
RÉSUMÉ
Le $\mathrm{XIX}^{\mathrm{e}}$ siècle voit apparaître une nouvelle vision de l'artiste. Face à une société bourgeoise et matérialiste, il s'érige en chantre d'un art hautain et désintéressé. Un tel écart entre l'artiste et le public doit produire des tensions et fait naître, des deux côtés, le sentiment d'incompréhension qui souvent dégénère en mépris. Charles Baudelaire contribue beaucoup à cette conception de l'art, où la notion de mépris revient souvent : le mépris envers le public bourgeois, l'artiste embourgeoisé, le critique d'art borné - mais aussi, le mépris du profanum uulgus dirigé contre l'artiste. Trois décennies plus tard, Octave Mirbeau fait appel à cette même notion pour construire son modèle des relations entre artiste et public, modèle bien proche de celui de Baudelaire. Notre objectif est donc double : analyser le concept du mépris chez les deux artistes, et relever les nombreuses affinités qui existent au niveau thématique, stylistique et rhétorique dans leurs écrits sur l'art.
\end{abstract}

MOTS-CLÉS - Mirbeau, Baudelaire, mépris, critique artistique

\section{"The Notion of Contempt in the Art Criticism of Baudelaire and Mirbeau"}

\begin{abstract}
SUMMARY
The nineteenth century sees a new vision of the artist. Faced with a bourgeois and materialistic society, he stands as a cantor of a haughty and disinterested art. Such a gap between the artist and the public must produce tension and give rise on both sides to the feeling of incomprehension which often degenerates into contempt. Charles Baudelaire contributes a lot to this conception of art, where the notion of contempt often comes up: contempt for the bourgeois public, the gentrified artist, the narrow-minded art critic - but also, the scorn of the profanum uulgus directed against the artist. Three decades later, Octave Mirbeau uses this same notion to build his model of artist - public relations, a model very similar to that of Baudelaire. Our objective is therefore twofold: to analyze the concept of contempt for both artists, and to identify many affinities that exist at the thematic, stylistic and rhetorical level in their writings on art.
\end{abstract}

KEYWORDS - Mirbeau, Baudelaire, contempt, art criticism 
En tâchant de préciser les « règles d'un genre $»^{1}$, J.-P. Leduc-Adine en déduit une, concernant le caractère forcément valorisant de la critique d'art : « dans le discours de la critique d'art, toute description est susceptible d'axiologisation, du fait de son investissement positif ou négatif: l'activité descriptive est toujours commentative $»^{2}$. Il développe cette idée notamment dans le contexte de la commercialisation de l'art, devenue la réalité du XIX ${ }^{e}$ siècle. «Dans ce système nouveau, écrit-il, l'œuvre d'art devient un placement, à propos duquel il convient de ne pas se tromper $»^{3}$. Le critique d'art serait donc un « médiateur » entre l'artiste et la clientèle potentielle, dont le rôle consisterait à expliquer l'œuvre, à influencer le goût du public, afin de garantir « la valeur - au sens monétaire du terme - de l'œuvre achetée $»^{4}$. Leduc-Adine n'hésite pas à voir dans le critique ainsi défini « un produit de la société bourgeoise, industrielle $»^{5}$. Or, à cette même époque, le concept de l'artiste toujours avide d'idéal et fier de son indépendance, s'oppose avec force à celui du bourgeois matérialiste et consumériste ${ }^{6}$. Que se passe-t-il donc lorsque l'artiste devient critique d'art ? Et, on le sait, au XIX ${ }^{\mathrm{e}}$ siècle, ces cas sont nombreux. Il semble que le fait d'être impliqué soi-même dans la création artistique doive influencer les jugements portés dans ce domaine. Si, toujours selon Leduc-Adine, la critique d'art se caractérise par l'utilisation de plusieurs procédés polémiques, à savoir « toute une série de marques énonciatives et de procédures rhétoriques, sémantiques et pragmatiques, qui ont toutes valeur disqualifiante vis-à-vis d'un artiste $»^{7}$, il paraît intéressant d'en extraire la notion de mépris, à cause de son caractère particulièrement incisif. Je voudrais étudier ses implications dans l'œuvre critique de deux artistes que sépare la distance de trente ans, mais que de nombreuses affinités rapprochent. En effet, même si Mirbeau a rarement cité Baudelaire dans ses écrits, l'influence du poète s'y fait sentir, comme d'ailleurs chez tant d'autres de sa génération. C'est le point de vue de Fabien Soldà, lorsqu'il cherche des analogies entre Les Fleurs du Mal et Le Jardin

\footnotetext{
J.-P. Leduc-Adine, « Des règles d'un genre : la critique d'art », Romantisme, 1991, n 71, p. 93-100.

2 Ibid., p. 93.

3 Ibid., p. 95.

4 Ibid.

5 Ibid.

6 Cette opposition, fréquente dans la littérature et l'art de l'époque, est également confirmée par des chercheurs. Gretchen van Slyke parle de " l'incompatibilité essentielle de l'artiste et du bourgeois $[\ldots]$ devenue un lieu commun [...] sous la monarchie de Juillet » ("Les épiciers au musée : Baudelaire et l'artiste bourgeois ", Romantisme, 1987, $\mathrm{n}^{\circ}$ 55, p. 55). Christophe Charle fait observer l'opposition qui existe, " dans l'imaginaire social, [entre] la vie d'artiste, la bohème, l'errance, l'incertitude, [le] travail improductif, [et] l'image tout aussi stéréotypée du 'bourgeois' et de la vie bourgeoise » (" Des artistes en bourgeoisie. Acteurs et actrices en Europe occidentale au XIX ${ }^{\mathrm{e}}$ siècle ", Revue d'histoire du XIX $X^{\mathrm{e}}$ siècle, 34 |2007, p. 72). Les analyses, devenues classiques, de Georges Matoré relèvent cette même opposition (« Le champ notionnel d'art et d'artiste entre 1827 et 1834 », in idem, La Méthode en lexicologie. Domaine français, Paris, Didier, 1973, p. 99-117).

7 J.-P. Leduc-Adine, op. cit., p. 99.
} 
des supplices ${ }^{8}$. Nous savons aussi que Mirbeau possédait dans sa bibliothèque deux éditions d'Euvres posthumes de Baudelaire, de 1887 et de 1908, ce deuxième sur hollande 9 . En ce qui concerne son œuvre de critique, des rapprochements sont également possibles, comme le signalent Pierre Miche ${ }^{10}$, Chantal Duverget ${ }^{11}$ ou Christian Limousin ${ }^{12}$. J'ai moi-même brièvement évoqué l'influence de Baudelaire sur Mirbeau dans ce domaine, dans un ouvrage traitant de son œuvre romanesque ${ }^{13}$.

\section{Dédain / mépris}

Baudelaire et Mirbeau ont une vision bien ample de l'art et de ses acteurs. Leur mépris se dirige, non seulement vers les manifestations d'un art médiocre, mais aussi vers ceux qui ne sont pas capables de le juger à sa valeur et qui, au contraire, nuisent à l'art digne de ce nom. Cependant, subissant dans leur propre chair des attaques injustes et l'incompréhension du public et de la critique, ils pourraient être tentés de manifester un dédain silencieux, tel le « dédain du juste » de Vigny ${ }^{14}$ ou le « arceo » d'Horace. D'autant que, hypersensibles tous les deux, ils se font assez tôt une piètre opinion de l'espèce humaine. La tour d'ivoire serait une solution en

8 Il cite Anatole Baju pour qui Baudelaire a été « le vrai précurseur [...] de l'école décadente » (F. Soldà, « Octave Mirbeau et Charles Baudelaire. Le Jardin des supplices ou Les Fleurs du Mal revisitées », Cahiers Octave Mirbeau n 4, 1997, p. 197).

9 Cf. J.-C. Delauney, « Mirbeau bibliophile, ou des clefs pour la bibliothèque d'Octave », et le tableau synoptique des ouvrages y appartenant, Cahiers Octave Mirbeau n ${ }^{\circ}$ 16, 2009, p. 31.

${ }^{10} \mathrm{Cf}$. le cinquième chapitre de sa thèse, intitulé « Le culte de l'art ou combats pour le beau ». P. Michel, Les Combats d'Octave Mirbeau, Annales littéraires de l'Université de Besançon, 1995, p. 125-158. Il fait voir également les parentés entre les deux artistes dans son article « Mirbeau et le symbolisme», Cahiers Octave Mirbeau n ${ }^{\circ}$ 2, 1995, p. 9-11.

${ }^{11}$ Elle observe que Besson situait Mirbeau «dans la lignée de Baudelaire », et le cite : «Et Mirbeau? L'alternance de ses dithyrambes et de ses pamphlets n'est-elle pas de la critique d'art? 'Partiale et politique...', comme la souhaitait Baudelaire ?» (Ch. Duverget, « George Besson. Compagnon de route d'Octave Mirbeau », Cahiers Octave Mirbeau n 20, p. 189. La citation de Besson provient de son article "Les écrivains et les arts visuels, de Diderot à Valéry », Les Lettres françaises $\mathrm{n}^{\circ}$ 840, 8 septembre 1960 , p. 10).

${ }^{12}$ Il rapproche les deux artistes sur le terrain d'une « critique d'invective » qui lui paraît les caractériser (Ch. Limousin, " Mirbeau critique d'art. De l' âge de l'huile diluvienne' au règne de l'artiste de génie », Cahiers Octave Mirbeau no 1, 1994, p. 15).

13 « Mirbeau est [...] profondément influencé par la conception baudelairienne de la critique : comme lui, il la veut partiale, imprégnée de la personnalité du critique et il érige l'émotion en critère central ». A. Staroń, L'Euvre romanesque d'Octave Mirbeau. Thèmes et techniques, Łódź, Wydawnictwo Uniwersytetu Łódzkiego, 2013, p. 157.

14 « Si le Ciel nous laissa comme un monde avorté, / Le juste opposera le dédain à l'absence / Et ne répondra plus que par un froid silence / Au silence éternel de la Divinité »; A. de Vigny, « Le Mont des Oliviers », 1862. 
somme logique. Or, ils se décident à prendre la parole ${ }^{15}$, et ils le font en recourant à la rhétorique où le mépris devient un outil de base.

Car il faut bien parler ici de mépris et le distinguer de dédain : d'abord, il semble que ce deuxième corresponde plutôt à une attitude distanciée, se caractérisant par un détachement. Le mépris, au contraire, s'extérioriserait davantage ${ }^{16}$. Arkadiusz Koselak insiste sur ce sens, en parlant, à propos du mépris, de «l'ordre de la monstration » et en lui attribuant une forte charge affective ${ }^{17}$. Empruntons-lui encore ces deux observations : $1^{\circ}$ "L'objet du mépris est contesté de façon plus radicale et abstraite, au sens où le jugement de valeur ne laisse aucun doute sur la négativité de ce qui est méprisé [...] le sujet s'arroge un pouvoir de discrimination éthique sur autrui qui est exorbitant »; $2^{\circ}$ " dédaigner c'est se détourner, faire un écart, se mettre de côté, tandis que mépriser c'est placer un objet (humain) en-dessous de, ce qui implique pour soi-même d'être au-dessus $»^{18}$. Ainsi, pour citer encore Koselak, « dédain, contrairement à mépris, résiste à la catégorisation, postulée, de sentiment $»^{19}$.

C'est rejoindre - et y répondre - les réflexions de Baudelaire adressées dans l'Envoi du Salon de 1859 aux « vrais artistes » qui « savent que rien n'est plus fatigant que d'expliquer ce que tout le monde devrait savoir. Si l'ennui et le mépris peuvent être considérés comme des passions, pour eux aussi le mépris et l'ennui ont été les passions les plus difficilement rejetables, les plus fatales, les plus sous la main $»^{20}$. C'est en même temps comprendre les raisons de ce mépris.

\section{Sources du mépris}

Elles gisent d'abord dans le contraste, consacré par la tradition romantique, entre les besoins du public bourgeois, ses goûts limités, et le raffinement de cet être sensible et supérieur qu'est un artiste. Elles découlent aussi des expériences propres de Baudelaire, Mirbeau et leurs confrères, avec une mauvaise critique qui ne comprend pas l'art et qui bafoue toute originalité. Il faut donc cerner les

${ }^{15}$ Les conditions premières de cette décision - à savoir la commande d'un journal, les besoins pécuniaires - ne paraissent pas changer l'essentiel, c'est-à-dire la manière dont ces relations sont conduites.

${ }^{16}$ Il suffit de regarder, à ce propos, la représentation graphique du mépris par Charles Le Brun, reproduite sur l'affiche de notre colloque : URL : https://i.pinimg.com/originals/29/78/e8/ 2978e8645a7b4a1f000f9adac5a8c947.jpg; consulté le 30.09.2018.

${ }^{17}$ A. Koselak, « Mépris / dédain, deux mots pour un même sentiment ? », Lidil [En ligne], 32 | 2005, p. 5 ; URL : http://lidil.revues.org/87 ; consulté le 30.09.2016.

${ }^{18}$ Ibid., p. 8.

${ }^{19}$ Ibid., p. 3.

${ }^{20}$ Ch. Baudelaire, Salon de 1859, Euvres complètes, Paris, Michel Lévy frères, 1868. Dans les citations ultérieures, l'abréviation S sera utilisée pour désigner les trois Salons de Baudelaire : S45, S46 et S59. 
principes artistiques de Baudelaire et de Mirbeau, afin de saisir les fondements de ce contraste, l'essence de ce conflit.

Les écrits sur l'art sont une bonne occasion, pour les deux artistes, de forger une esthétique, de mettre en mots ce qu'ils ressentent de manière intuitive. Cela vaut surtout pour Mirbeau qui n'a jamais formulé de doctrine complète, préférant lâcher par bribes ses idées sur l'art. Elles s'accordent en plusieurs points avec la pensée, plus construite, de Baudelaire. Ainsi, lorsque dans le fameux passage sur « la Reine des facultés », le poète déplore que le siècle moderne en vienne à « mépriser la plus honorable et la plus utile des facultés morales ", l'imagination (S59,9), sa manière de développer cette idée n'est pas sans rappeler les constatations de Mirbeau :

La nature n'est qu'un dictionnaire [...]. Les peintres qui obéissent à l'imagination cherchent dans leur dictionnaire les éléments qui s'accordent à leur conception ; encore, en les ajustant, avec un certain art, leur donnent-ils une physionomie toute nouvelle. Ceux qui n'ont pas d'imagination copient le dictionnaire. Il en résulte un très-grand vice, le vice de la banalité, qui est plus particulièrement propre à ceux d'entre les peintres que leur spécialité rapproche davantage de la nature extérieure, par exemple les paysagistes, qui généralement considèrent comme un triomphe de ne pas montrer leur personnalité. À force de contempler, ils oublient de sentir et de penser $(\mathrm{S} 59,10)$.

Pour Octave Mirbeau, « voir, sentir et comprendre $»^{21}$ sont une triade sans laquelle le livre de la nature reste fermé. Il est également convaincu de la nécessité de composer une œuvre d'art harmonieuse ${ }^{22}$, tout comme Baudelaire qui évoque « cette grande loi d'harmonie générale » $(\mathrm{S} 59,11)$.

La notion d'idéal, évidemment cruciale pour Baudelaire, est également analysée par Mirbeau. Dans son chapitre « De l'idéal et du modèle », Baudelaire, après avoir expliqué la contradiction du titre qui forme « un accord de contraires; car le dessin du grand dessinateur doit résumer l'idéal et le modèle » (S46, 24), nie l'existence de « l'idéal absolu » $(\mathrm{S} 46,25)$ et donne les conditions d'une exécution parfaite : il s'agit de comprendre l'essence d'un être ou d'un objet dessiné - pénétrer « les intentions de la nature »- et l'exprimer avec plus de relief et de clarté (S46, 26). Pareille pensée chez Mirbeau qui associe l'art véritable à « la recherche du vrai » et de l'idéal (CE1, 93), et souligne la nécessité d'un effort constant. Tout comme Baudelaire qui affirme : "Je m'impose à moi-même les dures conditions que je voudrais voir chacun s'imposer » (S59, envoi). Pour les deux, la synthèse est la condition de l'art (CE1, $93 ; \mathrm{S} 59,5)$.

${ }^{21}$ Cette formule revient plusieurs fois sous la plume de Mirbeau, notamment dans ses chroniques artistiques. On la retrouve aussi dans son roman Dans le ciel, Caen, L'Échoppe, 1989, p. 78.

${ }^{22}$ Qualité que Mirbeau invoque fréquemment, par exemple dans son article sur Camille Pissarro : «L'œil de l'artiste, comme sa pensée, découvre les grands aspects des choses, les totalités, l'harmonie ». Combats esthétiques 1, Paris, Séguier, 1993, p. 414. Pour les citations suivantes, j'utiliserai les abréviations CE1 et CE2. 
Tous les deux, en reconnaissant l'importance du « métier » chez un peintre, n'en font pas la chose la plus importante. Ainsi Baudelaire, en défendant la peinture de Corot, jugée imparfaite par quelques critiques, déclare qu'une œuvre « où tout est bien vu, bien observé, bien compris, bien imaginé - est toujours très bien exécutée, quand elle l'est suffisamment » $(\mathrm{S} 45,22)$. Mirbeau pardonne également des défauts d'exécution à un peintre qui sait par ailleurs comprendre la nature. En revanche, les deux sont sans pitié face à des dessinateurs habiles mais dépourvus de vision, des exécuteurs de reproductions photographiques ${ }^{23}$, tel Meissonier, bête noire de Mirbeau mais que Baudelaire n'épargne pas non plus (on y reviendra). Car ces artisans, souvent adulés par le public et par la critique, remplissent le rôle funeste de corrupteurs de goût national. Le public s'habitue à prendre la laideur pour de la vraie beauté, et passe indifférent (ou méprisant) à côté de véritables chefs-d'œuvre. Baudelaire repère ainsi, déjà à cette époque, une interaction présente d'autant plus de nos jours, entre l'artiste et le public :

Que l'artiste agisse sur le public, et que le public réagisse sur l'artiste, c'est une loi incontestable et irrésistible. [...] De jour en jour l'art diminue le respect de lui-même, se prosterne devant la réalité extérieure, et le peintre devient de plus en plus enclin à peindre, non pas ce qu'il rêve, mais ce qu'il voit $(\mathrm{S} 59,7)$.

\section{Cibles}

Le mépris de Baudelaire et de Mirbeau s'adresse à quelques cibles préférées. On peut les partager en trois groupes.

\subsection{Peintres médiocres}

En 1859, très déçu par la piètre qualité de l'exposition, Baudelaire les érige en représentants de l'art moderne dans sa totalité :

Que dans tous les temps la médiocrité ait dominé, cela est indubitable ; mais qu'elle règne plus que jamais, qu'elle devienne absolument triomphante et encombrante, c'est ce qui est aussi vrai qu'affligeant. [...] L'artiste, aujourd'hui et depuis de nombreuses années, est, malgré son absence de mérite, un simple enfant gâté. Que d'honneurs, que d'argent prodigués à des hommes sans âme et sans instruction ! [...] Discrédit de l'imagination, mépris du grand, amour (non, ce mot est trop beau), pratique exclusive du métier, telles sont, je crois, quant à l'artiste, les raisons principales de son abaissement (S59, 2-3).

${ }^{23}$ Auxquels Baudelaire consacre un long développement dans son Salon de 1859 pour conclure : « ce qui est positivement et universellement exact n'est jamais admirable» (S59, 14). Là également, il est secondé par Mirbeau qui « retrouve en M. Puvis de Chavannes [...] des sensations plus intenses et, par conséquent, plus nature que dans M. Roll, qui se borne à copier la nature, froidement, sans émotion, dans son apparence photographique et morte » $(\mathrm{CE} 1,305)$. 
Mirbeau déplore le manque de talent artistique en des termes bien proches. Et il parle également de médiocrité, qu'il appelle la moyenne :

En France, nous avons trois sources de richesses : les vins, les céréales et les arts; le phylloxéra détruit les vins, l'importation attaque les céréales, les arts sont rongés par la moyenne. La moyenne, c'est-à-dire ce qui flatte, ce qui caresse, ce qui réjouit l'âme bornée du public [...] la moyenne qui tortura Delacroix, Millet, Corot, la moyenne qui fait de Meissonier un génie [...]. Pour conquérir le succès, il faut au peintre, comme au littérateur, l'amour de la banalité compliquée, il doit avoir les qualités basses, et le vil esprit du vaudeville, la tristesse pleurnicheuse de la romance $(\mathrm{CE} 1,70)$.

Il est frappant à quel point les mêmes observations, les mêmes analyses reviennent chez les deux critiques-artistes.

Toute l'introduction du Salon de 1859 est méprisante pour les peintres qui y ont exposé. Baudelaire dénonce notamment leur manque de culture générale, d'imagination (qu'ils trouvent, dit-il, dangereuse) et de métier. Il en ressort un art bien plat et bien stéréotypé où " l'imitateur de l'imitateur trouve ses imitateurs 》 $(\mathrm{S} 59,4)$. La médiocrité de l'artiste moderne consisterait donc à manquer non seulement d'imagination, mais, fait encore plus grave, d'originalité : car il existe « une classe d'hommes, timides et obéissants, qui mettent tout leur orgueil à obéir à un code de fausse dignité » qui consiste à « élev[er] le poncif ${ }^{24}$ aux honneurs du style » $(\mathrm{S} 59,12)$.

\subsection{Critique bornée}

Une autre cible des deux artistes sont les critiques qui favorisent précisément ces peintres sans originalité. Baudelaire et Mirbeau affichent un violent mépris de leurs choix, goûts et opinions. Baudelaire parle ouvertement de la « bêtise des journalistes » $(\mathrm{S} 46,19)$ et de « la critique des journaux, tantôt niaise, tantôt furieuse, jamais indépendante » $(\mathrm{S} 45,1)$. « Ô critique ! ô critiques !...» (S45, 3), s'exclamet-il à l'occasion d'un commentaire sur Delacroix.

C'est également Delacroix ${ }^{25}$ qui fournit à Mirbeau le prétexte de cette opinion parfaitement désabusée de la critique :

Ce qu'il y a d'irrémédiable c'est qu'on ne peut même pas accuser la critique de mauvaise foi. Non. Elle n'a ni haine, ni parti-pris contre Delacroix. Elle n'a rien, et voilà ce qui est navrant, car elle ne voit et ne comprend rien. Elle pourrait écrire exactement le contraire, que cela ne tirerait pas, d'ailleurs, à conséquence (CE1, 138).

\footnotetext{
${ }^{24}$ Le poncif est, à côté du chic, le synonyme de l'art malsain et stéréotypé dans la pensée baudelairienne. Cf. S46, 32.

${ }^{25}$ À l'occasion d'une exposition au Palais des Beaux-Arts en 1885.
} 


\subsection{Le public}

Un chaînon autrement important de cette chaîne appelée art est le public, inculte et misonéiste dans sa majorité. L'espoir de pouvoir encore l'éduquer n'est que très léger. Ce public, dans le temps de Baudelaire et, à plus forte raison, celui de Mirbeau, se compose pour la plupart de bourgeois. Or, comme il a déjà été dit, l'antinomie entre bourgeois et artiste paraît un véritable topos du XIX $\mathrm{X}^{\mathrm{e}}$ siècle. Les passages des trois Salons, et surtout la fameuse Dédicace aux bourgeois du Salon 1846, semblent bien intéressants de ce point de vue. On y repère facilement l'ironie, mais cette dédicace manifeste-t-elle du mépris ? D'après les interprétations que font de ce texte Annie Becq et Gretchen van Slyke, Baudelaire ressent profondément l'ambiguïté de sa position, de bourgeois et d'artiste à la fois. Surtout dans les deux premiers Salons, il tâche de concilier son attitude de révolté avec la conscience que c'est bien le public bourgeois qui est destinataire de son art. Becq voit en lui un « antibourgeois qui pourtant ne se désolidarise pas de la bourgeoisie ${ }^{26}$ et, écartelé entre deux tendances opposées, il « s'associe au mépris des 'artistes', de règle depuis le Romantisme, pour le bourgeois » tout en vénérant « ces bourgeois qui ont depuis longtemps 'reconnu' l'art et ses vertus ». Effectivement, dans le salon de l'année précédente, Baudelaire précise ainsi son point de vue :

Et tout d'abord, à propos de cette impertinente appellation, le bourgeois, nous déclarons que nous ne partageons nullement les préjugés de nos grands confrères artistiques qui se sont évertués depuis plusieurs années à jeter l'anathème sur cet être inoffensif qui ne demanderait pas mieux que d'aimer la bonne peinture, si ces messieurs savaient la lui faire comprendre, et si les artistes la lui montraient plus souvent $(\mathrm{S} 45,1)$.

À ces questions idéologiques, Baudelaire ajoute un argument délibérément matériel : « le bourgeois [...] est fort respectable ; car il faut plaire à ceux aux frais de qui l'on veut vivre » $(\mathrm{S} 45,2)$. Et il déclare ouvertement ne pas suivre ceux qui méprisent le goût du large public :

Nous parlerons de tout ce qui attire les yeux de la foule et des artistes ; - la conscience de notre métier nous y oblige. - Tout ce qui plaît a une raison de plaire, et mépriser les attroupements de ceux qui s'égarent n'est pas le moyen de les ramener où ils devraient être $(S 45,2)$.

Il serait donc permis d'y découvrir la volonté d'éduquer ce public encore inculte. «Il était [...] du devoir de ce salonnier de censurer le dédain que les critiques prétentieux affichaient à l'égard de ceux qu'ils appelaient épiciers », constate G. van Slyke ${ }^{27}$.

\footnotetext{
${ }^{26}$ A. Becq, « Baudelaire et 'l'Amour de l'Art' : la dédicace 'Aux Bourgeois' du Salon de 1846 », Romantisme, 1977, $\mathrm{n}^{\circ}$ 17-18, p. 74.

${ }^{27}$ G. van Slyke, op. cit., p. 64.
} 
Il est vrai que nos critiques trouvent de temps en temps des mots plus doux envers les spectateurs. Baudelaire :

À mesure que le public voit de la bonne peinture, il se détache des artistes les plus populaires, s'ils ne peuvent plus lui donner la même quantité de plaisir. [...] le succès qu'obtient cette toile [de Jules Noël] prouve que, dans tous les genres, le public aujourd'hui est prêt à faire un aimable accueil à tous les noms nouveaux $(\mathrm{S} 46,42)$.

Mirbeau a de pareilles observations dans « La Nature et l'Art. À M. de Fourcaud», Gil Blas, 29 juin 1886 :

Le public a été depuis si longtemps dérouté par le dessin à contours fixes, par l'invariable représentation des formes convenues, par l'inflexibilité ennuyeuse et jolie des ensembles académiques, qu'il s'étonne bien un peu, n'y étant point habitué, dès qu'il se trouve en présence d'un coin de nature ou d'humanité recréé par un cerveau et par une main d'artiste (CE1, 304).

Mais avec le temps, les deux perdent leur élan et deviennent plus pessimistes, comme Baudelaire dans son Salon de 1859:

Or notre public, qui est singulièrement impuissant à sentir le bonheur de la rêverie ou de l'admiration (signe des petites âmes), veut être étonné par des moyens étrangers à l'art, et ses artistes obéissants se conforment à son goût ; ils veulent le frapper, le surprendre, le stupéfier par des stratagèmes indignes, parce qu'ils le savent incapable de s'extasier devant la tactique naturelle de l'art véritable $(\mathrm{S} 59,6)$.

Mirbeau ne voit pas non plus la possibilité de changer l'état des choses bien en place depuis des années :

je pense que dans les conditions morales, politiques et sociales où nous vivons, l'art ne peut être l'apanage que de quelques personnalités très rares et très hautes, affranchies de toute éducation officielle ou religieuse ; qu'il ne saurait être sensible au public, c'est-àdire à la masse sociale qui ne vit, ne pense, n'agit d'après la loi des conventions arbitraires et du mensonge, et qu'avant de donner une éducation artistique au public, il faudrait l'y préparer par le long et impossible enseignement de la vérité... si tant est qu'il y ait une vérité dans la vie et que nous la connaissions » (CE2, 338-339).

D'autant que tous les deux observent, à la fois chez le public et chez les artistes français, certains traits inamovibles :

Dans le sens le plus généralement adopté, Français veut dire vaudevilliste, et vaudevilliste un homme à qui Michel-Ange donne le vertige et que Delacroix remplit d'une stupeur bestiale, comme le tonnerre certains animaux. Tout ce qui est abîme, soit en haut, soit en bas, le fait fuir prudemment. Le sublime lui fait toujours l'effet d'une émeute, et il n'aborde même son Molière qu'en tremblant et par ce qu'on lui a persuadé que c'était un auteur gai $(\mathrm{S} 46,33)$. 
L'idée sur l'esprit vaudevilliste français sera reprise par Mirbeau entre autres dans son « Claude Monet» :

Nous sommes vraiment de misérables gamins, destructeurs inconscients du beau et du vrai, à qui toute grandeur, toute éloquence, toute sincérité échappent. Peuple de blagueurs, multitude grimaçante de cabots, nous n'aimons que l'exagération du mot, le grincement bête du rire, le drapement théâtral des douleurs. Il faut que nous voyions toutes choses à travers des cinquièmes actes de vaudeville et de mélodrame et que nous forcions la nature et la vie à se plier à toutes les déformations de l'esprit - esprit de concierge et de chroniqueur $(\mathrm{CE} 1,82)$.

Les remarques sur l'esprit français - ou encore celui de vaudevilliste abondent dans la critique de nos deux artistes, qui le dénoncent comme responsable de la superficialité de jugement et du mauvais goût des compatriotes. « Si l'artiste abêtit le public, celui-ci le lui rend bien », observe Baudelaire (S59, 5). Mirbeau dénonce les railleries du public confronté à un art plus difficile d'accès, car s'écartant des routes tracées par les écoles officielles. Maintes fois, il s’indigne contre « les insultes » et « les blagues canailles » prodiguées à ces artistes sincères et - à cause de cela - « honnis et $[\ldots]$ méprisés » $(\mathrm{CE} 1,69)$.

\section{Formes de mépris}

Les citations précédentes ont déjà conduit vers le dernier point de cette réflexion: les moyens utilisés pour traduire le mépris. Je voudrais y distinguer deux types primordiaux :

D'abord, un mépris affiché et correspondant tout à fait à sa définition. Il est froid, sérieux, ne cache pas sa volonté de blesser, comme dans cette critique acerbe que Baudelaire donne, non seulement du peintre, mais encore de la ville et de son esprit bourgeois :

On devine trop, en regardant ce tableau cru et luisant, que M. Janmot est de Lyon. En effet, c'est bien là la peinture qui convient à cette ville de comptoirs, ville bigote et méticuleuse, où tout, jusqu'à la religion, doit avoir la netteté calligraphique d'un registre $(\mathrm{S} 46,28)$.

Baudelaire n'a pas envie de plaisanter. Il lance toute crue son opinion impitoyable, destinée à écraser, ici les disciples maladroits d'Ingres :

Du reste, toutes ces afféteries passeront comme des onguents rancis. Il suffit d'un rayon de soleil pour en développer toute la puanteur. J'aime mieux laisser le temps faire son affaire que de perdre le mien à vous expliquer toutes les mesquineries de ce pauvre genre $(\mathrm{S} 46,29)$.

Mais plus souvent il exprime son mépris par une forme légère, ironique. Seulement parfois plus grinçant, la plupart du temps il veut amuser le lecteur, 
et lui montrer, par là même, la distance et l'air hautain qui devraient caractériser les artistes dignes de ce nom ${ }^{28}$. Car en utilisant l'ironie, le critique se place en position supérieure par rapport à son sujet, et en le plaisantant, il lui signifie son mépris.

Ainsi, faut-il bien voir une forme de mépris dans cette façon amusante et, somme toute, négligente de traiter ce peintre dont Baudelaire décrit les tableaux et les compétences. On dirait que Diaz de la Peña ne sait pas la moindre chose de la technique picturale. C'est le traiter avec condescendance ${ }^{29}$ :

M. Diaz de la Peña [...] part de ce principe qu'une palette est un tableau. Quant à l'harmonie générale, M. Diaz pense qu'on la rencontre toujours. [...] M. Diaz est coloriste, il est vrai ; mais élargissez le cadre d'un pied, et les forces lui manquent, parce qu'il ne connaît pas la nécessité d'une couleur générale. C'est pourquoi ses tableaux ne laissent pas de souvenir $(\mathrm{S} 46,23)$.

Ce type de commentaires abonde dans les trois Salons. Simon Saint-Jean en souffre peut-être plus souvent que les autres : «Au total, quelque bien faits qu'ils soient, les tableaux de M. Saint-Jean sont des tableaux de salle à manger, - mais non des peintures de cabinet et de galerie ; de vrais tableaux de salle à manger » $(\mathrm{S} 45,25)$, constate Baudelaire.

Nos deux critiques ont quelques figures de choix qu'ils décrivent dans leurs textes. Meissonier, peintre historique militaire captive l'attention de Baudelaire : «M. Meissonier exécute admirablement ses petites figures. C'est un Flamand moins la fantaisie, le charme, la couleur et la naïveté - et la pipe !» $(\mathrm{S} 45,21)$ Dans le Salon de 1859, Baudelaire revient à ce peintre, avec plus de malice :

Il est bon de hausser la voix et de crier haro sur la bêtise contemporaine, quand, à la même époque où un ravissant tableau de Delacroix trouvait difficilement acheteur à mille francs, les figures imperceptibles de Meissonier se faisaient payer dix fois et vingt fois plus. Mais ces beaux temps sont passés ; nous sommes tombés plus bas, et M. Meissonier, qui, malgré tous ses mérites, eut le malheur d'introduire et de populariser le goût du petit, est un véritable géant auprès des faiseurs de babioles actuels (S59, 3).

Mirbeau est infiniment plus cruel envers Meissonier à qui il fait plusieurs fois allusion dans ses chroniques. Il lui consacre aussi des articles à part, comme

${ }^{28}$ Que cela n'ait pas toujours été vrai - est une autre histoire. Évidemment, ils se sentaient blessés, ils étaient parfois résignés et abattus, ils perdaient l'espoir dans l'amélioration du goût public. C'est bien visible dans les trois Salons de Baudelaire ; c'est encore visible dans les Combats, toujours plus aigris, de Mirbeau. Mais leur engagement découle, avant tout, de la nécessité de formuler ses propres opinions esthétiques, et, comme tel, il doit impliquer l'émotivité. Le mépris est donc, d'un côté, un outil commode de cette propagande, mais, de l'autre, une arme pour protéger sa propre sensibilité. Il faudrait y ajouter, dans le cas de Baudelaire, son attitude de dandy, méprisante et distanciée faute de place, je me limite à signaler cette tendance, évidemment absente chez Mirbeau.

${ }^{29}$ Qui est voisine du mépris. Cf. URL : http://www.cnrtl.fr/definition/condescendance ; consulté le 30.09.2018. 
« Votons pour Meissonier» où il invite les lecteurs à appuyer la candidature du peintre comme sénateur, convaincu que son manque de talent en peinture fera son succès en politique. Le commentaire de l'art du peintre que Mirbeau construit par la même occasion est aussi parmi les plus méprisants :

Je ne sais rien de plus ennuyeux qu'un tableau de M. Meissonier, parce qu'il y manque toujours cette qualité maîtresse de l'art : la vie. Jamais M. Meissonier n'a été ému par le spectacle d'un être ou d'une chose. [...] Tout est sacrifié à une virtuosité mécanique, ennuyeuse et fatigante, à la recherche d'un détail puéril, seulement visible à la loupe et qui n'importe pas. Qu'est-ce que cela me fait que M. Meissonier sache mieux qu'un capitaine d'habillement la longueur d'une capote de fantassin et le numéro d'un schako d'artilleur? Quelle conscience, s'écrie-t-on, quelle exactitude! (CE1, 232)

Cependant Baudelaire a aussi sa bête noire, Horace Vernet, chez qui il relève exactement les mêmes défauts que ceux notés par Mirbeau. La mise en parallèle de ces deux passages révèle des similitudes jusqu'au choix des mêmes mots :

Du reste, pour remplir sa mission officielle, M. Horace Vernet est doué de deux qualités éminentes, l'une en moins, l'autre en plus : nulle passion et une mémoire d'almanach ! Qui sait mieux que lui combien il y a de boutons dans chaque uniforme, quelle tournure prend une guêtre ou une chaussure avachie par des étapes nombreuses; à quel endroit des buffleteries le cuivre des armes dépose son ton vert-de-gris ? Aussi, quel immense public et quelle joie ! Autant de publics qu'il faut de métiers différents pour fabriquer des habits, des shakos, des sabres, des fusils et des canons ! Et toutes ces corporations réunies devant un Horace Vernet par l'amour commun de la gloire ! Quel spectacle ! (S46, 33)

Des exemples de ce type sont beaucoup plus nombreux chez Mirbeau; d'abord, à cause de son tempérament qui le porte bien plus loin que Baudelaire à manifester son dégoût de la médiocrité, mais aussi, tout simplement, à cause d'un nombre infiniment plus grand d'écrits qu'il a consacrés à l'art ${ }^{30}$. Relevons un article parmi d'autres, à cause de sa violence particulière qui dépasse les bornes de la convenance, s'attaquant à un défunt. Dans " Oraison funèbre », Mirbeau parle d'Alexandre Cabanel qui était « médiocre, médiocre immensément, médiocre avec passion, avec rage, avec férocité» et pour cela «se montrait impitoyable au génie ». «Sij'avais une épitaphe à inscrire sur sa tombe, je mettrais simplement ceci : 'Ci-gît un professeur : Il professa' » (CE1, 351, 353). La cruauté est ici corollaire de l'immense mépris qui émane de chaque mot. Et pourtant, l'effet humoristique est également obtenu.

Un pareil résultat - un mépris écrasant sous une forme amusante - peut être obtenu à l'aide des phrases courtes, cinglantes, qui à elles seules constituent toute l'appréciation du tableau :

${ }^{30}$ Les deux volumes des Combats esthétiques comptent à eux seuls 180 chroniques, auxquelles il faut ajouter les « Premières chroniques esthétiques », les écrits sur la musique et les articles consacrés à la littérature. 
Jacquand

Fabrique toujours du Delaroche, vingtième qualité.

Roehn

Peinture aimable (argot de marchand de tableaux) (S45, 21).

Une telle technique est très efficace : ces phrases courtes sont comme un coup d'œil rapide qui suffit pour se convaincre du manque d'intérêt du tableau et pour formuler une opinion méprisante par sa brièveté même. Ainsi Mirbeau en uset-il avec délices :

M. Maurice Leloir. - Parfumerie, ganterie. - La meilleure pâte épilatoire se vend à la parfumerie Leloir. - Eau de toilette Leloir rafraîchit le teint et resserre les tissus de la peau. - Teinture sans acide. - Poudre de riz de toutes marques. (Demandez le prospectus.)

Mme Madeleine Lemaire. - Fleurs et plumes. - Mannequins pour couturières.

M. Worms. - Grand déballage de castagnettes. - Lot de guitares. - Solde défraîchi de mantilles (CE1, 114).

C'est assener à ces peintres un coup violent - mais qui émane précisément d'un profond mépris.

Enfin, le mépris peut s'exprimer par des phrases quelque peu paradoxales, mais qui se laissent facilement comprendre. Ainsi de cette critique de Félix Haffner qui avait d'abord causé une impression favorable sur Baudelaire ; mais ce charme fut vite dissipé : « je croyais que c'était un grand artiste plein de poésie et surtout d'invention, un portraitiste de premier ordre [...] ; mais il paraît que ce n'est qu'un peintre » (S46, 24). Il apparaît alors que Haffner n'a pas compris l'art ; il n'est qu'un artisan, dans la mauvaise acception du mot. Telle phrase de Mirbeau fait pendant, une fois de plus, aux propos de Baudelaire : «Un peintre qui n'a été qu'un peintre ne sera jamais que la moitié d'un artiste » $(\mathrm{CE} 1,143)$.

Ce parcours nécessairement rapide à travers les écrits sur l'art de Baudelaire et de Mirbeau a permis, me semble-t-il, de relever de nombreuses affinités entre l'esthétique et la manière de concevoir l'art de ces deux artistes. Il a surtout montré des analogies dans leur critique - passionnée et individualiste, pleine d'admiration pour des artistes dignes de ce nom et impitoyable pour ceux qui ne le méritent pas. Déjà en 1846, Baudelaire déclarait : «pour être juste, c'est-à-dire pour avoir sa raison d'être, la critique doit être partiale, passionnée, politique, c'est-à-dire faite à un point de vue exclusif » $(\mathrm{S} 46,3)$. Le mépris paraît une conséquence naturelle d'une telle prise de position. Les deux artistes s'unissent dans le mépris pour les critiques de profession, s'il est permis d'étendre cette appellation aux journalistes responsables des rubriques sur l'art dans les journaux et les revues. Baudelaire et Mirbeau ne se sentent pas solidaires de ce groupe, pour la raison évidente qu'ils sont eux-mêmes artistes et comprennent donc le sentiment et les objectifs de l'autre bord. De plus, dotés d'une sensibilité et d'un talent extraordinaires, ils sont capables, selon leur propre définition, de « voir, sentir et comprendre » davantage. Cela élargit le fonctionnement de la notion de mépris à plusieurs autres occurrences : 
en dehors de la critique injuste et aveugle, c'est également le mépris de nos deux artistes envers le public borné, envers les artistes peu doués ou arrivistes ; mais c'est aussi le mépris de ce public envers les œuvres et les artistes qu'il n'est pas capable de comprendre ${ }^{31}$; et le mépris de ladite critique pour ces artistes originaux, qui échappent à sa vision restreinte de l'art.

Si Baudelaire et Mirbeau recourent avec une telle régularité au mépris, c'est qu'il est pour eux un commode outil d'expression. Ainsi, faut-il distinguer entre mépris didactique qui charrie l'espoir de former encore le public ; mépris purement esthétique, leur réaction à des phénomènes qui leur déplaisent et les incitent à le manifester, même sans grand espoir d'influencer le spectateur ; enfin mépris envers ceux qui contribuent à fausser le goût public : il se lie à la conscience qu'il s'agit là, le plus souvent, de choix mercantiles, purement opportunistes.

Il ne faut pas non plus négliger le mépris, possible à détecter, envers le profanum uulgus, cette masse inculte et qui n'aspire pas à s'éduquer, car elle est convaincue de sa force et de sa position dominante - et qui offense la sensibilité des deux artistes.

En somme, le mépris de Baudelaire et de Mirbeau est certes une forme de critique d'art, mais aussi un moyen de présenter les éléments de leur propre esthétique. Tout en méprisant, ils s'engagent et ils luttent; il s'agit bien d'une implication polémique qui demande de l'activité, mais en même temps vise à former le goût des lecteurs à venir. Le temps a montré que la plupart de leurs luttes ont été gagnées. Cela ne fait que confirmer l'efficacité de leur méthode.

\section{Bibliographie}

\section{Sources}

Baudelaire, Charles, Salon de 1845, Salon de 1846 et Salon de 1859, in Euvres complètes de Charles Baudelaire, Michel Lévy frères, 1868 :

URL : https://fr.wikisource.org/wiki/Salon_de_1845; consulté le 30.09.2016

URL : https://fr.wikisource.org/wiki/Salon_de_1846; consulté le 30.09.2016

URL : https://fr.wikisource.org/wiki/Salon_de_1859; consulté le 30.09.2016

Mirbeau, Octave, Combats esthétiques, t. 1 et 2, édition établie, présentée et annotée par Pierre Michel et Jean-François Nivet, Paris, Librairie Séguier, 1993

Mirbeau, Octave, Dans le ciel, Caen, L’Échoppe, 1989

${ }^{31}$ Rodin, dans la relation de Mirbeau, s'étonne que « des gens qui se disent des artistes, mettent tant de passion aveugle et méchante » contre son art honnête, fait " de conscience, de respect et de sacrificies » (CE1, 117). Mais c'est surtout l'article « La Tristesse de M. Boulanger » qui est un véritable concert de froid mépris envers ce peintre, professeur à l'École des Beaux-Arts, qui sans se limiter à ignorer les mérites de l'art impressionniste, ose encore les vilipender. La réponse de Mirbeau serait toute à citer, pleine de flèches habiles dirigées vers l'académicien et dénigrant l'importance du Salon; que cette phrase nous en suffise : « Les petites gens à qui, du haut de vos académies vermoulues, de votre chaire inécoutée, vous jetez froidement l'injure, se sont appelés jadis Velasquez et Rembrandt ; ils s'appelaient hier Millet et Corot, ils s'appellent aujourd'hui Puvis de Chavannes, Manet, Degas, Claude Monet et Renoir » (CE1, 152-153). 


\section{Études}

Becq, Annie, « Baudelaire et 'l'Amour de l'Art' : la dédicace 'Aux Bourgeois' du Salon de 1846 », Romantisme, 1977, no 17-18. Le bourgeois, p. 71-78 ; URL : https://www.persee.fr/doc/ roman_0048-8593_1977_num_7_17_5125; consulté le 30.09.2016

Bourrelier, Pierre-Henri, " Octave Mirbeau et l'art au début du XX ${ }^{\mathrm{e}}$ siècle », Cahiers Octave Mirbeau, $\mathrm{n}^{\circ} 10,2003$, p. 167-185

Charle, Christophe, « Des artistes en bourgeoisie. Acteurs et actrices en Europe occidentale au XIX siècle ", Revue d'histoire du XIX' ${ }^{\mathrm{e}}$ siècle, 34 | 2007, p. 71-104, URL : http://journals.openedition. org/rh19/1322 ; DOI : https://doi.org/10.4000/rh19.1322; consulté le 5.01.2021

Delauney, Jean-Claude, « Mirbeau bibliophile, ou des clefs pour la bibliothèque d'Octave », Cahiers Octave Mirbeau n ${ }^{\circ}$ 16, Angers, 2009, p. 119-165

Duverget, Chantal, " George Besson. Compagnon de route d'Octave Mirbeau », Cahiers Octave Mirbeau $\mathrm{n}^{\circ}$ 20, p. 187-189

Koselak, Arkadiusz, « Mépris / dédain, deux mots pour un même sentiment ? », Lidil 32 | 2005, mis en ligne le 05 octobre 2007 ; URL : http://lidil.revues.org/87 ; consulté le 30.09.2016

Leduc-Adine, Jean-Pierre, «Des règles d'un genre : la critique d'art », Romantisme, 1991, n 71. Critique et art, p. 93-100, URL: https://www.persee.fr/doc/roman_0048-8593_1991_num_21_71_5737; consulté le 30.09.2016

Limousin, Christian, "Mirbeau critique d'art. De l' âge de l'huile diluvienne' au règne de l'artiste de génie », Cahiers Octave Mirbeau n 1, 1994, p. 11-41

Matoré, Georges, «Le champ notionnel d'art et d'artiste entre 1827 et 1834 », in idem, La Méthode en lexicologie. Domaine français, Paris, Didier, 1973, p. 99-117

Michel, Pierre, Les Combats d'Octave Mirbeau, Annales littéraires de l'Université de Besançon, 1995

Michel, Pierre, « Mirbeau et le symbolisme », Cahiers Octave Mirbeau n 2, 1995, p. 8-22

Slyke, Gretchen, van, «Les épiciers au musée : Baudelaire et l'artiste bourgeois », Romantisme, $1987, \mathrm{n}^{\circ} 55$

L'artiste, l'écrivain, le poète, p. 55-66 ; URL : https://www.persee.fr/doc/roman_0048-8593_1987_ num_17_55_4861; consulté le 30.09.2016

Soldà, Fabien, « Octave Mirbeau et Charles Baudelaire. Le Jardin des supplices ou Les Fleurs du Mal revisitées », Cahiers Octave Mirbeau no 4, 1997, p. 197-216

Staroń, Anita, L'Euvre romanesque d'Octave Mirbeau. Thèmes et techniques, Łódź, Wydawnictwo Uniwersytetu Łódzkiego, 2013

Vigny, Alfred, de, «Le Mont des Oliviers », Les Destinées, Paris, Michel Lévy frères, 1864

Anita Staroń, HDR, travaille à l'Institut d'études romanes de l'Université de Łódź. Elle enseigne la littérature française du XIX ${ }^{\mathrm{e}}$ siècle. Son domaine de recherche est le roman français de la fin du XIX et du début du XX $\mathrm{XX}^{\mathrm{e}}$ siècle, avec un intérêt particulier pour l'œuvre d'Octave Mirbeau et de Rachilde. C'est à ces deux auteurs que sont consacrées ses monographies : L'Art romanesque d'Octave Mirbeau. Thèmes et techniques, Łódź, Wydawnictwo Uniwersytetu Łódzkiego, 2013 ; et Au carrefour des esthétiques. Rachilde et son écriture romanesque. 1880-1913, Łódź, Wydawnictwo Uniwersytetu Łódzkiego, 2015.

\begin{tabular}{|l|l|}
\hline cc Creative \\
common & $\begin{array}{l}\text { C by the author, licensee Łódź University - Łódź University Press, } \\
\text { Łódź, Poland. This article is an open access article distributed under } \\
\text { the terms and conditions of the Creative Commons Attribution license } \\
\text { CC-BY-NC-ND 4.0 (https://creativecommons.org/licenses/by-nc-nd/4.0/) }\end{array}$ \\
\cline { 2 - 2 } & Received: 2019-01-15; Accepted: 2020-11-08 \\
\hline
\end{tabular}

